\title{
Ciberacoso escolar: experiencias y propuestas de jóvenes universitarios
}

\section{(Cyberbullying: experiences and proposals of university students)}

\author{
Juan Carlos Rivadulla López \\ Universidade da Coruña, UDC (España) \\ Marisol Rodríguez Correa \\ Universidad Internacional de la Rioja, UNIR (España)
}

DOI: http://dx.doi.org/10.5944/ried.22.2.23541

\section{Cómo referenciar este artículo:}

Rivadulla López J. C., y Rodríguez Correa, M. (2019). Ciberacoso escolar: experienciasy propuestas dejóvenes universitarios. RIED. Revista Iberoamericana de Educación a Distancia, 22(2), pp. 179-201. doi: http://dx.doi.org/10.5944/ ried.22.2.23541

\section{Resumen}

El rápido crecimiento de las TIC ha traído consigo múltiples beneficios, pero, sin duda alguna, también muchos perjuicios, siendo uno de ellos el ciberacoso, el cual está tomando cada día más fuerza en nuestra sociedad. El interés principal en este estudio fue conocer la percepción y experiencia de un grupo de jóvenes universitarios sobre el ciberacoso en su etapa estudiantil en el instituto de educación secundaria. Para la realización de esta investigación, hemos contado con la participación de 864 estudiantes de seis universidades del noreste de España. Se ha utilizado una metodología cuantitativa de carácter no experimental de tipo encuesta, concretamente se utilizó un muestreo no probabilístico, accidental o incidental, condicionado por la disponibilidad de los sujetos a participar en el estudio. La mayoría del alumnado consultado en nuestro estudio indicó no haber sufrido ciberacoso ni haberlo ejercido en su etapa estudiantil en el Instituto tanto en centros públicos como privados. La agresividad escrita (insultos/ofensas o amenazas) fue el tipo de acción violenta más habitual entre los agresores, al igual que la difusión de información personal o de rumores. La mayoría de jóvenes, que sufrieron acoso en el Instituto en nuestro estudio, reconocieron que no se sintieron motivados para acudir a clase en su momento, ni para desarrollar sus estudios de manera adecuada, aislándose socialmente.

Palabras clave: tecnología; ciberacoso; educación; estudiantes. 


\begin{abstract}
The rapid growth of ICT has brought many benefits, but certainly also many damages, one of them being cyberbullying, which is taking more and more strength in our society. The main interest in this research was to know the perception and experience of a group of young university students about cyberbullying in their student stage at the secondary education context. To carry out this research, we had the participation of 864 students of six universities in the northeast of Spain. A quantitative methodology of non-experimental type character survey has been used and, in particular, sampling non-probability has been used, accidental or incidental, conditioned by the availability of subjects to participate in this research. Most of the students in our study indicated not having suffered ciberbullying or exercising it in his student stage both in state and private schools. Written aggression (insults / offenses or threats) was the most common type of violent action among the aggressors, and the dissemination of personal information or rumors. The majority of young people, who suffered harassment in the school in our study, acknowledged that they did not feel motivated to attend sessions at the time, nor to develop their studies in an adequate way, isolating themselves socially.
\end{abstract}

Keywords: technology; cyberbullying; education; students.

El acoso escolar y ciberacoso han adquirido en la actualidad una mayor importancia, debido a que cada vez se da en edades más tempranas y de forma más alarmante (Moral, Suárez, Villarreal y Musitu, 2014); y, si bien es cierto que en todas las épocas en las Instituciones Educativas han existido alumnos con comportamientos disruptivos y actitudes inadecuadas, tal y como señala Marchena (2012), en los últimos años las mismas se vienen enfrentando a situaciones de acoso escolar y ciberacoso cada vez más graves.

Según Garaigordobil (2011), el acoso escolar afecta a un 20\% y 30\% de estudiantes a nivel nacional e internacional, siendo el porcentaje de victimización grave entre el 3 y el 10\% y, en el caso del ciberacoso, los resultados arrojados en el estudio reciente realizado por Zych, Ortega-Ruiz y Marín-López (2016) permiten concluir que la prevalencia media es del $26.65 \%$, con un rango desde un $78.31 \%$ (experiencias ocasionales) hasta un $4.60 \%$ (ciberacoso severo).

Investigaciones llevadas a cabo en los últimos años en los centros escolares españoles indican en sus resultados que hay menores que presentan dificultad en la integración escolar y en el proceso de aprendizaje porque afirman tener miedo y sentirse indefensos ante la amenaza, la violencia y el dominio de su agresor (Muñoz, 2016). Por ello, se ha establecido una reglamentación para proteger al menor de los delitos de tipo cibernético, desarrollada en el artículo 197 del Código Penal y modificado en las Leyes Orgánicas 5/2010 de 22 de junio, 3/2011 de 28 de enero y 1/2015 de 30 de marzo (Fundación Mutua Madrileña y la Fundación Anar, 2016).

Cuando hablamos de ciberacoso, por su parte, nos referimos a los procesos relacionados con el acoso a través de los dispositivos digitales y entornos virtuales. Sin 
duda alguna, la incorporación de las TIC en la vida cotidiana de nuestras sociedades ha generado una cultura de lo virtual, en la cual se han incorporado nuestros niños y jóvenes. De esta forma, la tecnología permite, por una parte, transmitir formas de pensar, de ser, de comportarse y, por la otra, ha dado lugar a nuevas estructuras de agresión entre iguales, como es el caso del ciberacoso o cyberbullying (Smith, Mahdavi, Carvallo, Fisher, Russel y Tippet, 2008; Slonje, Smith y Frisén, 2013).

Las conductas poco seguras en Internet, el uso de redes sociales y de programas de mensajería instantánea, la frecuencia de uso de Internet y la falta de control parental son algunos factores de riesgo tanto de cibervictimización ocasional como habitual (Álvarez-García, Núñez, Dobarro y Rodríguez, 2015).

Algunas de las formas más comunes a través de las cuales se manifiesta el ciberacoso son las amenazas y burlas, la suplantación de la identidad y el envío de material con contenido sexual, siendo el Internet y el teléfono móvil los principales medios utilizados por los jóvenes para hacerlo (Calvete, Orue, Estévez, Villardón, y Padilla, 2010; Garaigordobil y Aliri, 2013; Durán-Segura y Martínez-Pecino, 2015).

Consideramos que no podemos concebir el acoso escolar y ciberacoso como fenómenos separados, ya que lo que sucede a través de los medios digitales forma parte de la misma forma de la realidad cotidiana de niños y jóvenes, lo que ocurre es que a través de la tecnología se extiende el acoso escolar hasta el hogar u otros ámbitos, no siendo necesario tener a la víctima en frente para humillarla y hacerla sentir mal.

Estudios realizados sobre el tema indican que las víctimas de acoso y ciberacoso experimentan una falta de aceptación entre sus iguales, lo que conlleva en la mayoría de los casos a la baja autoestima, la depresión y el aislamiento social (Perren, Dooley, Shaw y Cross, 2010; Sourander, BrunsteinKlomek, Ikonen, Lindroos, Luntamo, Koskelainen, Ristkari y Helenius, 2010; Zwierzynska, Wolke y Lereya, 2013). Otros estudios, como el realizado por García-Bacete, Sureda y Monjas (2010), señalan que los adolescentes víctimas carecen de habilidades interpersonales eficaces, incidiendo esto negativamente en su ajuste personal, escolar y social, lo que produce un incremento de su vulnerabilidad para ser acosados y ciberacosados por sus pares (Rodríguez-Hidalgo, Ortega-Ruiz y Zych, 2014; Navarro, Ruiz-Oliva, Larrañaga y Yubero, 2015).

Tal y como señalan García y García (2015), las políticas públicas han de tomar en cuenta que el Sistema Educativo, además de ser un instrumento de transmisión de conocimientos, es un medio de socialización, un vehículo de transferencia de valores y un recurso social de interacción personal en el que los problemas de convivencia surgen frecuentemente como en cualquier tipo de relación social.

La puesta en práctica de programas de intervención en donde cooperen profesores, padres y estudiantes ayudará a combatir el acoso y ciberacoso en todas sus formas de expresión. Dichos programas deberán ser planteados según Garaigordobil y Oñederra (2010) en cuatro niveles: 
1. Institucional: todo el centro educativo debe estar implicado.

2. Familiar: es fundamental la participación de los padres en el proceso, informando de los resultados de las evaluaciones que se llevan a cabo en el centro y solicitando su colaboración y seguimiento cuando se producen incidentes.

3. Grupal: llevando a cabo programas de intervención socio-emocional y antibullying en las aulas de clase.

4. Individual: con el agresor y con la víctima.

Por otra parte, es importante destacar que una propuesta de intervención en el acoso y el ciberacoso deberá incluir: (1) Prevención (actuaciones dirigidas a mejorar la convivencia, prevenir la conflictividad y evitar la aparición del fenómeno); (2) Intervención primaria (cuando se detectan situaciones de acoso incipientes, para evitar su consolidación, a través de la aplicación de un programa específico con intervenciones individuales y con el grupo de estudiantes, etc.); e 3) Intervención secundaria (cuando se trata de situaciones consolidadas, dirigida a minimizar el impacto sobre los implicados aportando apoyo terapéutico y protección a las víctimas, así como control a los agresores) (Garaigordobil, Martínez y Machimbarrena, 2017).

Si bien es cierto que en los últimos años se han realizado muchos estudios acerca del acoso o ciberacoso en estudiantes de Secundaria (Avilés, 2006; León, Felipe, Fajardo y Gómez, 2012; Palacios, Polo, Felipe, León y Fajardo, 2013; Del Rey, Durán-Segura y Martínez-Pecino, 2018; Garaigordobil, 2015; Cerezo, Arnaiz, Giménez y Maquilón, 2016; Garaigordobil, Martínez y Machimbarrena, 2017; Gimenez, Arnaiz, Cerezo-Ramírez y Prodócimo, 2018; Mora-Merchán, Casas, Ortega-Ruiz y Elipe, 2018; Yubero, Larrañaga, Navarro y Elche, 2018), son menos las investigaciones realizadas sobre el fenómeno del acoso o ciberacoso en estudiantes universitarios. Por este motivo, pretendemos realizar un estudio con un grupo de alumnos universitarios para conocer sus experiencias de ciberacoso, ya que consideramos importante conocer la vivencia y opinión de estos jóvenes que formarán parte de nuestro estudio, pues se trata de uno de los sectores poblacionales que mayoritariamente se ha podido ver involucrado en esta problemática.

\section{MÉTODO}

El interés principal en este estudio fue conocer la percepción y experiencia de un grupo de jóvenes universitarios sobre el ciberacoso en su etapa estudiantil en el Instituto, entendido como una forma específica de violencia escolar, donde uno o varios agresores con mayor poder y con intencionalidad causan dolor, acosan y someten reiteradamente a un compañero/a.

Teniendo en cuenta el contenido que aborda este estudio y, por motivos de protección de datos, se prefiere mantener en el anonimato el nombre de las universidades donde se llevó a cabo la investigación, indicando que la recogida de datos se realizó a principios del curso académico 2016/2017 de forma presencial 
y en formato papel en seis universidades del noroeste de España. Para ello, se contactó con los responsables de las instituciones educativas para obtener el permiso necesario y, posteriormente, con los docentes que tendrían que permitir acudir a sus clases para que su alumnado cubriera el cuestionario. Cabe destacar que, en algunos casos, fueron los propios docentes (ajenos a la investigación) los que distribuyeron el cuestionario, lo cual nos facilitó el trabajo a los investigadores. Los cuestionarios se devolvieron de la misma forma que lo recibieron (en formato papel y de forma presencial).

La participación fue voluntaria y se garantizó el anonimato y la confidencialidad de los datos obtenidos. Para responderlo, se les concedió el tiempo que ellos consideraron oportuno, teniendo una duración media de 30 minutos.

\section{Participantes}

Para la realización de esta investigación, hemos contado con la participación de 864 estudiantes, de los cuales la mayoría son mujeres $(70,83 \%)$ de entre 18 y 20 años (55,09\%), estudian algún grado relacionado con las Ciencias Sociales y Jurídicas $(36,23 \%)$ y la mayoría viven en un entorno urbano $(60,76 \%)$. Además, todos los participantes disponen de teléfono móvil, así como conexión a Internet desde casa y desde el móvil (ver tabla 1).

Tabla 1. Distribución de los participantes

\begin{tabular}{|c|c|c|c|}
\hline & & $\mathbf{F}$ & $\%$ \\
\hline \multirow{6}{*}{$\begin{array}{l}\text { Rama de } \\
\text { conocimiento }\end{array}$} & Artes y humanidades & 106 & 12,27 \\
\hline & Ciencias & 227 & 26,27 \\
\hline & Ciencias de la salud & 129 & 14,93 \\
\hline & Ciencias sociales y jurídicas & 313 & 36,23 \\
\hline & Ingeniería y Arquitectura & 89 & 10,30 \\
\hline & TOTAL & 864 & 100 \\
\hline \multirow{3}{*}{ Sexo } & Hombres & 252 & 29,17 \\
\hline & Mujeres & 612 & 70,83 \\
\hline & TOTAL & 864 & $10 O$ \\
\hline \multirow{5}{*}{ Edad } & De 18 a 20 años & 476 & 55,09 \\
\hline & De 21 a 23 años & 221 & 25,58 \\
\hline & De 24 a 26 años & 99 & 11,46 \\
\hline & Mayores de 27 años & 68 & 7,87 \\
\hline & TOTAL & 864 & 100 \\
\hline
\end{tabular}




\begin{tabular}{|c|c|c|c|}
\hline & & $\mathbf{F}$ & $\%$ \\
\hline \multirow{3}{*}{$\begin{array}{l}\text { Lugar de } \\
\text { residencia }\end{array}$} & Urbano & 525 & 60,76 \\
\hline & Rural & 339 & 39,24 \\
\hline & TOTAL & 864 & 100 \\
\hline \multirow{6}{*}{$\begin{array}{l}\text { Recursos } \\
\text { tecnológicos }\end{array}$} & Dispone de teléfono móvil & 864 & 100 \\
\hline & TOTAL & 864 & 100 \\
\hline & Dispone de Internet en casa & 864 & 100 \\
\hline & TOTAL & 864 & 100 \\
\hline & Dispone de Internet en el móvil & 864 & 100 \\
\hline & TOTAL & 864 & 100 \\
\hline
\end{tabular}

\section{Instrumento}

Se realizó una adaptación del cuestionario propuesto por Ortega, Calmaestra y Mora-Merchán (2007), el cual mide las relaciones entre los jóvenes a través de las nuevas tecnologías (Teléfono Móvil e Internet), así como también el acoso que dicen sufrir o ejercer dichos jóvenes a través de estas tecnologías.

Los apartados, que incluyó el cuestionario, se elaboraron en torno al objetivo que nos marcamos para esta investigación y, por supuesto, teniendo en cuenta toda la fundamentación teórica expuesta anteriormente. Este cuestionario fue revisado por tres expertos en la materia, con el fin de validar su contenido y su inteligibilidad para el participante, realizando las modificaciones oportunas. Teniendo en cuenta las correcciones realizadas, se aplicó el instrumento a una muestra piloto de 25 estudiantes ( 5 de cada rama de conocimiento), con la finalidad de detectar problemas de funcionamiento de los ítems antes de su aplicación a la totalidad de la muestra (Expósito, Navarro, Thoilliez y López, 2010), pero el resultado fue óptimo y no se necesitó realizar más cambios.

La estructura del instrumento está organizada alrededor de cuatro grandes dimensiones que hay que tener en consideración en el análisis de este problema, incluyéndose en cada una de ellas una serie de preguntas abiertas y/o cerradas (ver anexo): a) Casos de ciberacoso observados en el Instituto (1 pregunta); b) Perfil de los participantes que admiten haber sufrido ciberacoso en el Instituto ( 5 preguntas); c) Perfil de los participantes que admiten haber ejercido ciberacoso en el Instituto (5 preguntas); d) Medidas contra el ciberacoso (2 preguntas). A mayores, se han incluido 8 preguntas de identificación (edad, sexo, rama de conocimiento, etc.).

\section{Procedimiento de análisis}

Para dar respuesta a la intencionalidad exploratoria y descriptiva de este estudio (Bisquerra, 2004), se ha utilizado una metodología cuantitativa de carácter no 
experimental de tipo encuesta (McMillan y Schumacher, 2005). Concretamente, se utilizó un muestreo no probabilístico, accidental o incidental, condicionado por la disponibilidad de los sujetos a participar en el estudio. Este tipo de muestreos, aunque no permiten «extraer muestras representativas de la población, sí facilitan el estudio cualitativo en profundidad del tema que interesa dentro de un contexto determinado» (Martínez, 2007, p. 56). Como condiciones para poder colaborar en este estudio, nos propusimos que los participantes utilizaran habitualmente Internet (tanto desde el PC como desde el móvil). El análisis de las respuestas recogidas en el cuestionario se analizó de forma directa, estableciéndose porcentajes, y fue realizado independientemente por dos investigadores, discutiendo las posibles discrepancias. Además, se aplicó estadística descriptiva y la prueba estadística de independencia de criterios Chi-cuadrado con 95\% de confianza para determinar la asociación entre las variables de interés (sexo, tipo de centro educativo y lugar de residencia), y las categorías de ciberacoso sufrido y ejercido. Este análisis estadístico se realizó con el programa SPSS 24.

Para elaborar y sintetizar los resultados, lo primero que se hizo fue identificar el número de personas que indicaron haber sufrido acoso a través del móvil o de Internet (apartado a). A continuación, y tomando como referente los cuatro grandes apartados que se incluyen en el cuestionario, se procedió a analizar las respuestas a las preguntas: a) Identificación de los participantes en cuanto al ciberacoso sufrido y ejercido; b) los casos de ciberacoso que los participantes observaron en el instituto (pero que ellos no eran víctimas); c) el perfil de los participantes que admiten haber sufrido ciberacoso en el Instituto; d) el perfil de los participantes que admiten haber ejercido ciberacoso en el Instituto; y e) las medidas que se tomaron en su etapa estudiantil ante el ciberacoso y las que se deberían tomar según ellos.

\section{RESULTADOS}

\section{Identificación de los participantes en cuanto al ciberacoso sufrido y ejercido}

En general, los datos muestran que pocos son los participantes que confirman haber sufrido ciberacoso y, aún menos, los que indican haberlo ejercido (tabla 2). No obstante, entre los que indican que han sufrido o ejercido ciberacoso se puede establecer un tipo de perfil atendiendo a la mayoría porcentual, siendo este en ambos casos el de una mujer que estudiaba en un centro público cuando se produjo el acoso y que vive en un entorno urbano.

Los resultados muestran que existen diferencias significativas entre el ciberacoso sufrido y el ejercido por los participantes y el sexo $(\mathrm{p}=0,002$ y $\mathrm{p}=0,000$ respectivamente), el tipo de centro educativo ( $\mathrm{p}=0,012 \mathrm{y} \mathrm{p}=0,001)$ y el lugar de residencia $(\mathrm{p}=0,001$ y $\mathrm{p}=0,000)$. 
Tabla 2. Frecuencia/porcentaje de participantes $(n=864)$ y Chi Cuadrado de Pearson $\left(\chi^{2}\right.$, $\mathrm{p}<0,05)$ en relación al acoso sufrido y al ejercido a través de Internet

\begin{tabular}{|c|c|c|c|c|c|c|c|}
\hline & & \multicolumn{3}{|c|}{ Ciberacoso sufrido } & \multicolumn{3}{|c|}{ Ciberacoso ejercido } \\
\hline & & Sí & No & $x^{2}$ & Sí & No & $x^{2}$ \\
\hline \multirow{2}{*}{ Sexo } & Hombres & $\begin{array}{c}90 \\
(10,4)\end{array}$ & $\begin{array}{c}162 \\
(18,8)\end{array}$ & \multirow{2}{*}{,002 } & $\begin{array}{c}54 \\
(6,3) \\
\end{array}$ & $\begin{array}{c}198 \\
(22,9)\end{array}$ & \multirow{2}{*}{, OOO } \\
\hline & Mujeres & $\begin{array}{c}237 \\
(27,4)\end{array}$ & $\begin{array}{c}375 \\
(43,4)\end{array}$ & & $\begin{array}{c}126 \\
(14,6)\end{array}$ & $\begin{array}{c}486 \\
(56,3)\end{array}$ & \\
\hline \multirow{2}{*}{$\begin{array}{l}\text { Tipo de Centro } \\
\text { Educativo }\end{array}$} & Público & $\begin{array}{c}205 \\
(23,7)\end{array}$ & $\begin{array}{c}305 \\
(35,3)\end{array}$ & \multirow{2}{*}{,012 } & $\begin{array}{c}108 \\
(12,5)\end{array}$ & $\begin{array}{c}402 \\
(46,5)\end{array}$ & \multirow{2}{*}{,001 } \\
\hline & Privado-Concertado & $\begin{array}{c}122 \\
(14,1)\end{array}$ & $\begin{array}{c}232 \\
(26,9)\end{array}$ & & $\begin{array}{c}72 \\
(8,3) \\
\end{array}$ & $\begin{array}{c}282 \\
(32,6)\end{array}$ & \\
\hline \multirow{2}{*}{$\begin{array}{l}\text { Lugar de } \\
\text { residencia }\end{array}$} & Urbano & $\begin{array}{c}230 \\
(26,6) \\
\end{array}$ & $\begin{array}{c}295 \\
(34,1) \\
\end{array}$ & \multirow{2}{*}{,001 } & $\begin{array}{c}121 \\
(14,0)\end{array}$ & $\begin{array}{c}404 \\
(46,8) \\
\end{array}$ & \multirow{2}{*}{, oOO } \\
\hline & Rural & $\begin{array}{c}97 \\
(11,2)\end{array}$ & $\begin{array}{c}242 \\
(28,0)\end{array}$ & & $\begin{array}{c}59 \\
(6,8) \\
\end{array}$ & $\begin{array}{c}280 \\
(32,4)\end{array}$ & \\
\hline
\end{tabular}

\section{Casos de ciberacoso observados en el Instituto}

En cuanto al número de casos de acoso observados por los participantes en su Instituto (ya sea acoso presencial o ciberacoso), apenas existen diferencias entre el alumnado de centros públicos y de centros privados (figura 1). Concretamente, la mayoría indica que nunca fueron testigos presenciales del mismo $(34,5 \%$ de centros públicos y 38,2\% de centros privados). Entre el alumnado que sí presenció acoso, la mayoría, tanto de centros públicos como privados, revela que este solo ha sucedido una o dos veces (33,7\% y $26,5 \%$ respectivamente). 
Figura 1. Casos de acoso observados en el instituto

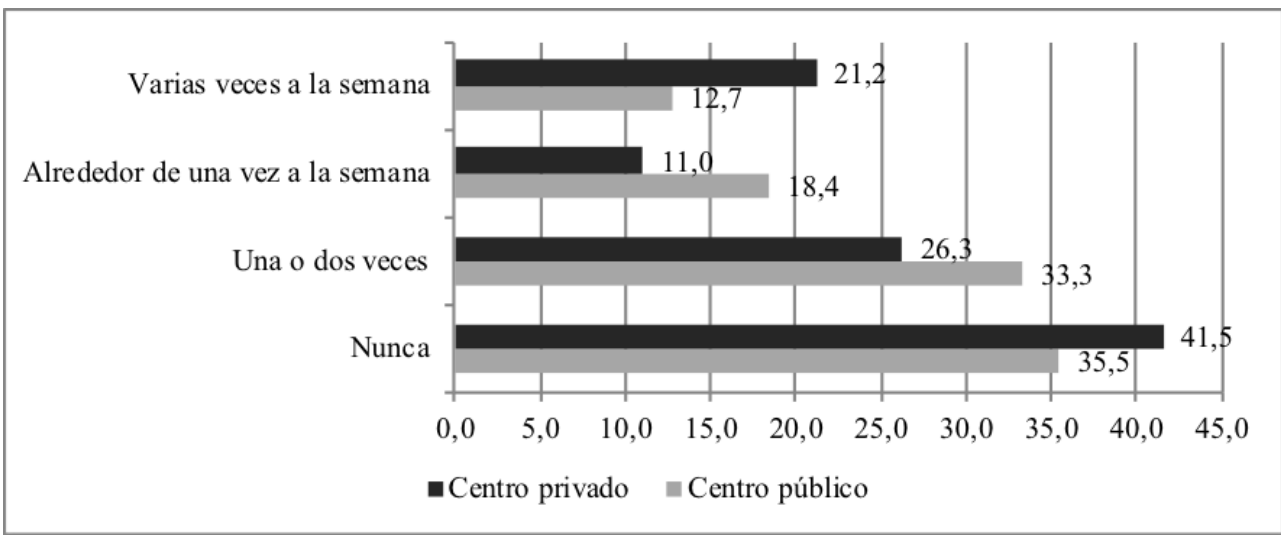

\section{Perfil de los participantes que admiten haber sufrido ciberacoso en el Instituto}

A la hora de establecer un perfil de persona acosada (figura 2), existen diferencias entre hombres y mujeres respecto a la edad, ya que los hombres empezaron a sufrir ciberacoso escolar entre los 13 y los 15 años $(47,8 \%)$, mientras que en las mujeres esa edad asciende hasta los 16-17 años (37,1\%).

Con respecto a las veces que los participantes han sufrido ciberacoso, la mayoría (más del 60\%) indica que nunca lo han sufrido ni ejercido. De los estudiantes que indican que sí lo han sufrido, la mayoría revela que este hecho se ha producido durante varias veces a la semana (15,2\% de mujeres y $14,3 \%$ de hombres).

En cuanto al tipo de ciberacoso sufrido, la agresividad escrita (insultos/ofensas o amenazas) es el tipo de acción violenta más habitual entre los agresores, tanto para hombres como para mujeres (en más del 70\% de todos los casos). La difusión de información personal o de rumores es un hecho que también sufrieron entre el $40 \%$ y $60 \%$ de hombres y mujeres. De igual forma, es bastante frecuente tanto el aislamiento social, el cual se percibe más en el entorno femenino $(41,8 \%)$ que en el masculino (31,1\%), como la exclusión en redes sociales, cuyos valores difieren de lo anterior (21,1\% en mujeres y $24,4 \%$ en hombres). El resto de actos (compartir sin permiso fotos y vídeos comprometidos o suplantar la identidad de una persona en redes sociales), aunque no menos importantes, presentan un nivel de incidencia inferior al $20 \%$ en todos los casos. 
Figura 2. Edad de inicio, frecuencia y tipo de ciberacoso sufrido

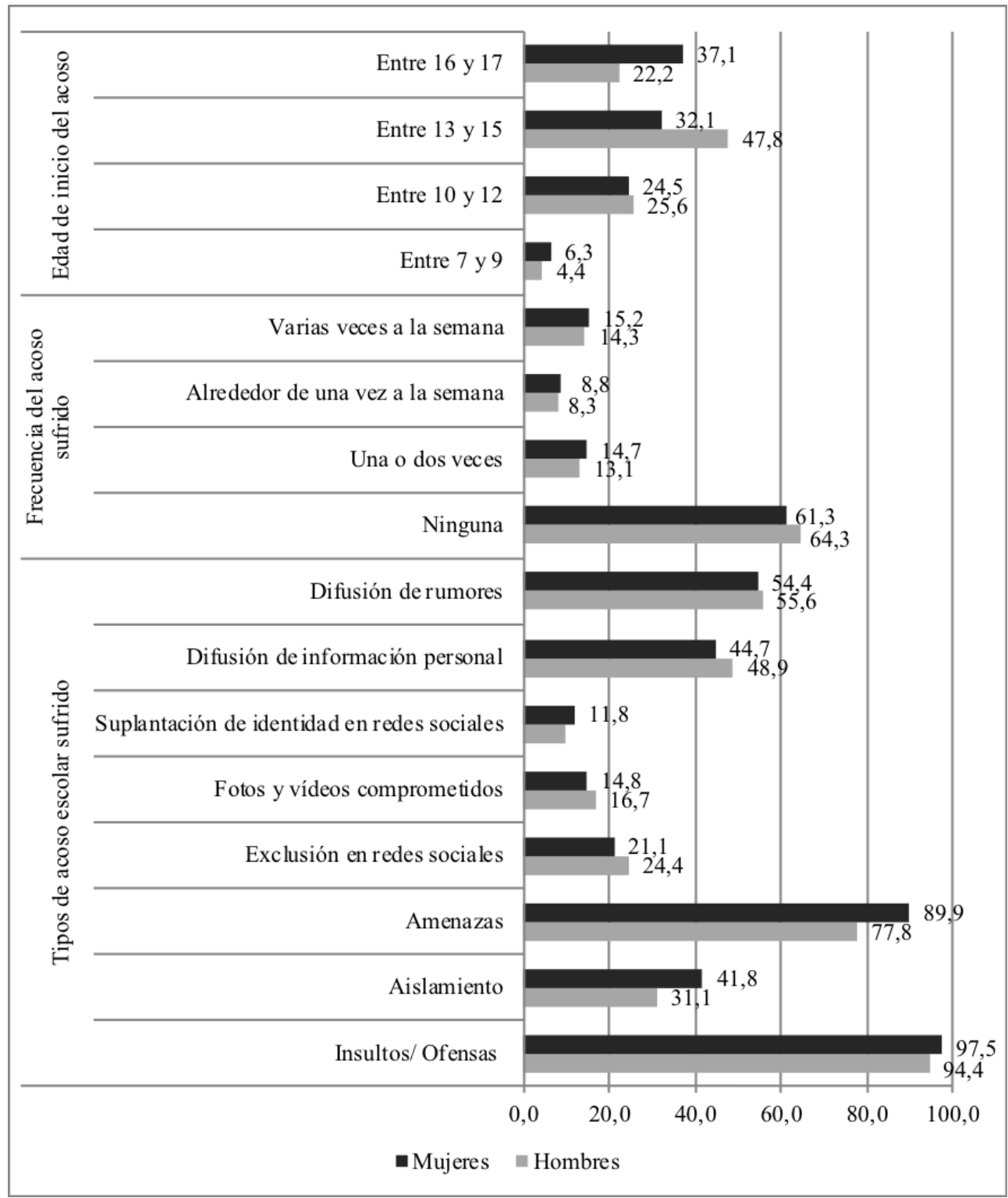

En cuanto al sentimiento que tuvieron mientras sufrían acoso (figura 2), la mayoría de las mujeres indica que cuando les ciberacosaron han respondido a los 
acosadores por el mismo medio (chat, whatsapp, redes sociales) $(40,5 \%)$ y han llorado $(34,2 \%)$. Además, confiesan que se lo comunicaron a sus amigos $(38,8 \%)$, pero esa comunicación no fue tan fluida cuando se trata de padres $(24,5 \%)$ o docentes (17,3\%) (figura 3). En cuanto a los hombres, mayoritariamente indican que han llorado $(24,2 \%)$ y se lo han comunicado a los docentes $(24,4 \%)$.

En relación con la satisfacción de las víctimas en el Instituto mientras sufrían acoso, la inmensa mayoría reconoce que tenía una satisfacción baja pues no se sentía motivado/a para acudir a clase ni, mucho menos, para desarrollar sus estudios de manera adecuada (52,7\% de mujeres y $60 \%$ de hombres).

Figura 3. Sentimiento y satisfacción escolar ante el ciberacoso sufrido

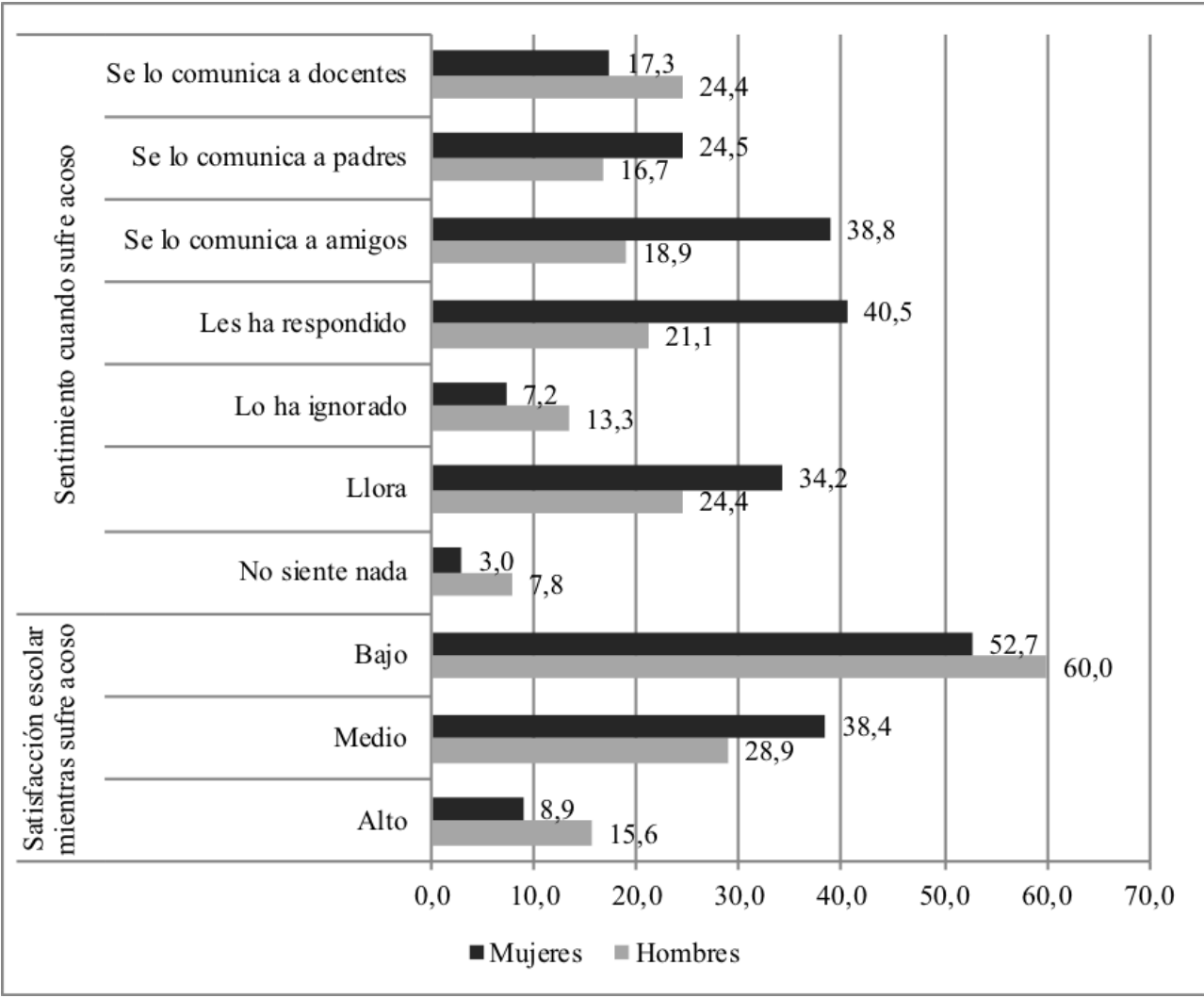




\section{Perfil de los participantes que admiten haber ejercido ciberacoso en el Instituto}

De los participantes que indicaron que ejercieron ciberacoso escolar durante su etapa en el Instituto (figura 4), existen diferencias entre hombres y mujeres respecto a la edad, ya que los hombres empezaron a ejercerlo de forma mayoritaria entre los 13 y los 15 años $(51,9 \%)$, mientras que en las mujeres esa edad asciende hasta los 16-17 años (39,7\%). En cuanto a la frecuencia que tuvo ese ciberacoso, la igualdad entre hombres y mujeres es abrumadora, ya que ambos colectivos se decantaron por indicar que fue ocasional y duró entre una y dos veces (57,1\% las mujeres y $66,7 \%$ los hombres).

Por su parte, un porcentaje notable de las víctimas mujeres en ciberacoso tiene entre sus agresores a antiguos amigos/as (45,2\%), mientras que, en el caso de los hombres, los agresores indican que nunca habían sido amigos de las víctimas (66,7\%).

En referencia al número de personas que participaban en el acoso, más del 50\% de los participantes, tanto hombres como mujeres, indicaron que lo hacían entre dos y cinco personas (55,6\%), aunque los agresores solitarios también forman una categoría importante, ya que se corresponden con un 20,6\% de las mujeres y un $27,8 \%$ en los hombres.

En cuanto a los motivos principales del ciberacoso, estos se encuentran en las características diferenciadoras de las víctimas y en la diversión y agresividad de los acosadores. Concretamente, ambos colectivos tienen en cuenta las características físicas de la víctima (peso, gafas, etc.) (38,9\% en el caso de los agresores hombres y $30,2 \%$ en el caso de las mujeres) o simplemente el hecho de querer hacer una broma (48,1\% de los hombres y 43,7\% de las mujeres). Cabe destacar que un porcentaje importante de hombres $(31,5 \%)$ también indicó que había ciberagredido a compañeros debido a la orientación sexual de estos. 
Figura 4. Perfil del acosador

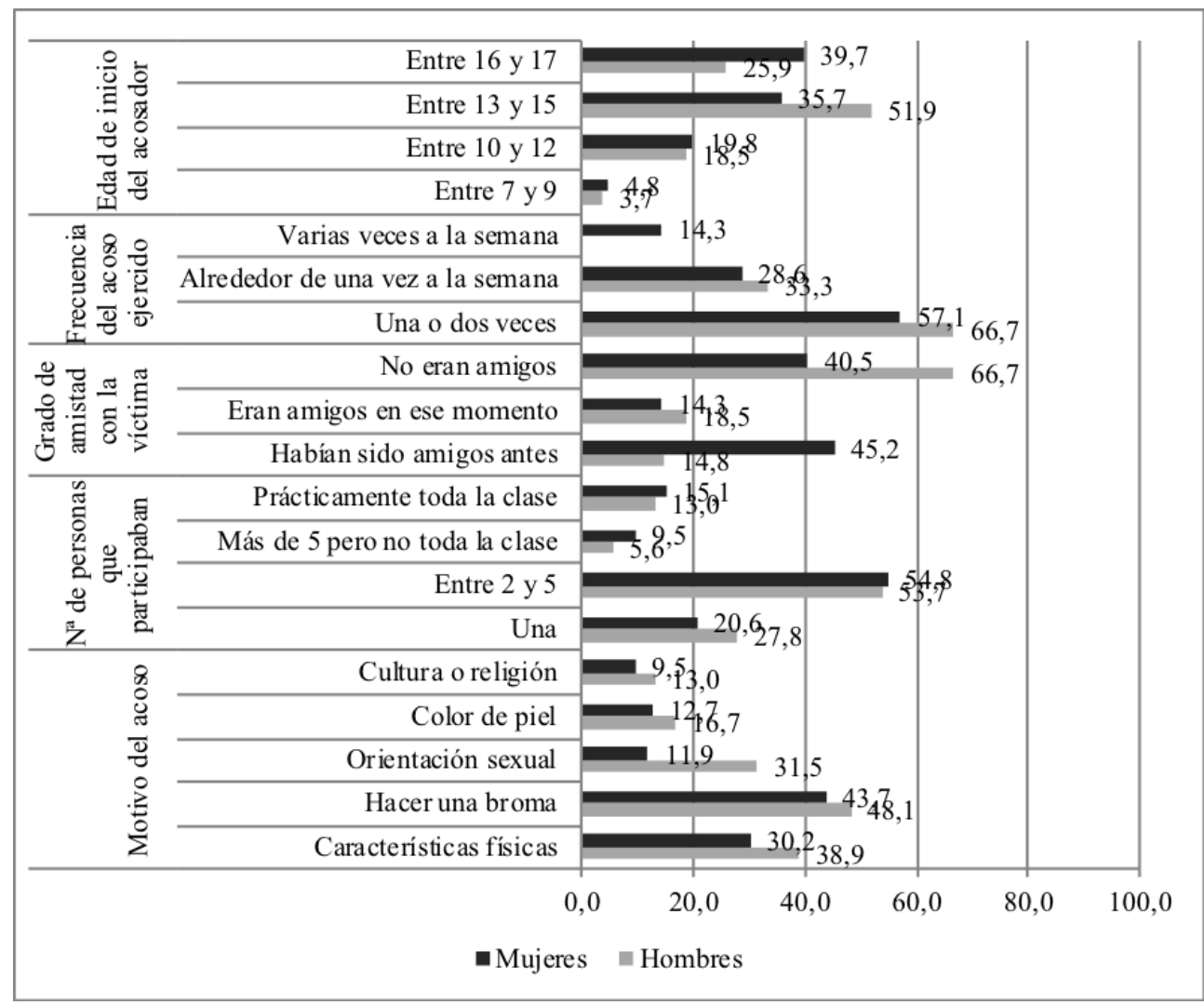

\section{Medidas contra el ciberacoso}

Respecto a la actuación que los participantes recuerdan que se llevaba a cabo en su Instituto ante casos de ciberacoso (figura 5), la mayoría de los que pertenecían a centros públicos indica que se sancionaba al alumno acosador $(44,1 \%)$ o que se ignoraba el hecho sucedido (41,0\%), mientras que los del centro privado se decantan por la opción de la sanción de manera mayoritaria (50\%). En menor medida, alumnos de ambos tipos de centros también se refieren a que hablaban del tema en clase $(22,4$ públicos y $32,2 \%$ privados).

Por otra parte, respecto a las propuestas de actuación ante casos de ciberacoso que los participantes plantean, la mayoría de los que estudiaron, tanto en centros públicos como en privados, se decantan por la necesidad de formar al profesorado (90,4\% y 80,5\% respectivamente) y a las familias $(80,4 \%$ y 70,3\%), para que estén 
atentos a cambios repentinos en los adolescentes, manteniendo una relación de confianza con ellos para que, en caso de problemas, sepan a quienes pueden acudir. Entre el 60\% y el 70\% de los participantes de ambos colectivos, también indican que las escuelas y las familias deben trabajar coordinadamente, por lo que las administraciones educativas deben ofrecerles las herramientas legislativas y las indicaciones para la prevención e intervención efectivas en temas relacionados con el ciberacoso, estableciendo protocolos de actuación a nivel nacional/autonómico. Por último, el alumnado perteneciente a centros públicos se refiere en mayor medida a crear conciencia de las consecuencias que acarrea el ciberacoso $(79,4 \%)$ y a promover habilidades sociales como la empatía o la asertividad, etc. $(34,3 \%)$, mientras que más del 50\% de los participantes de centros privados se refieren a la importancia de cuidar la privacidad (emplear cuentas privadas en redes sociales, limitar el número de contactos, configurar opciones de privacidad, no compartir información sensible, etc.).

Figura 5. Medidas ante el ciberacoso explicitadas por los participantes

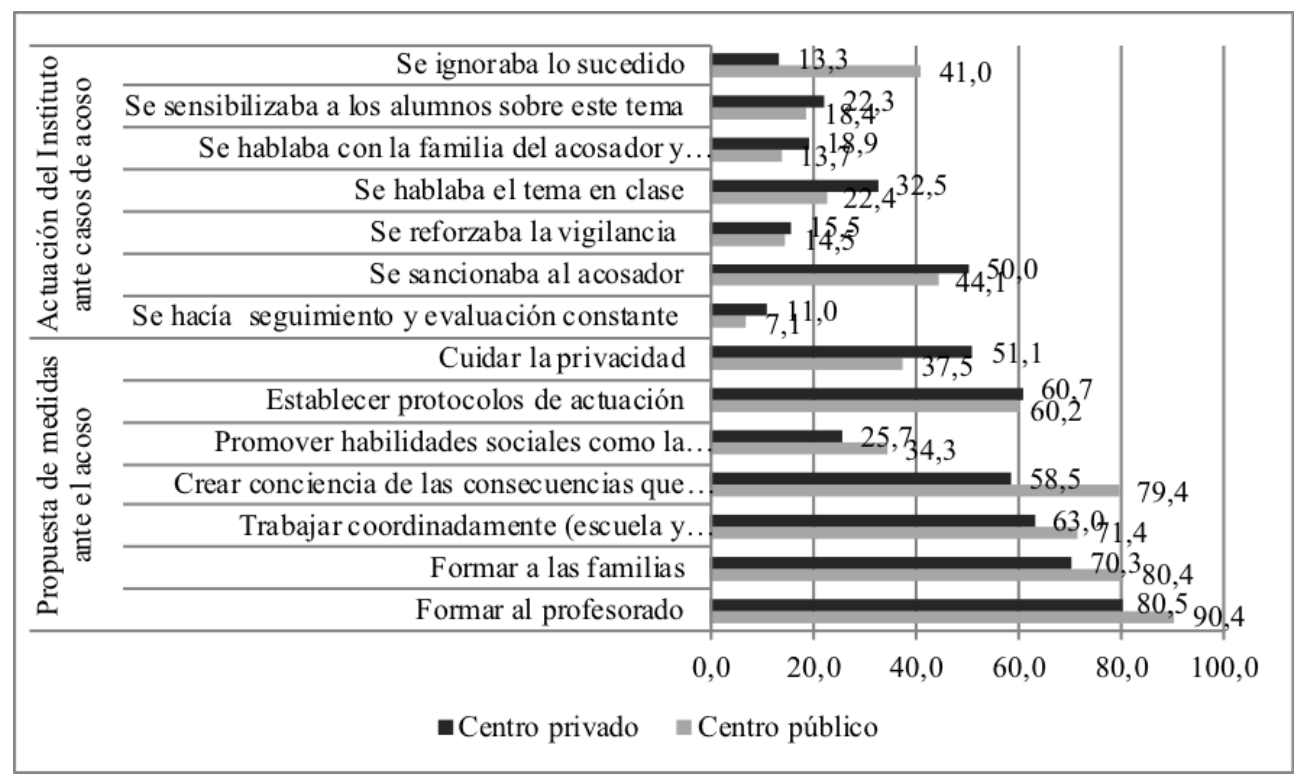

\section{DISCUSIÓN}

Con nuestro estudio, hemos pretendido conocer la percepción y experiencia de un grupo de jóvenes universitarios sobre el ciberacoso en su etapa estudiantil del 
Instituto. De los resultados obtenidos, podemos extraer una serie de reflexiones que nos permiten situarnos con mayor conocimiento ante el tema estudiado:

- La mayoría del alumnado consultado en nuestro estudio indicó no haber sufrido ciberacoso ni haberlo ejercido en su etapa estudiantil en el Instituto tanto en centros públicos como privados. De los estudiantes que indicaron que sí lo han sufrido, la mayoría reveló que este hecho se ha producido durante varias veces a la semana.

- La agresividad escrita (insultos/ofensas o amenazas) fue el tipo de acción violenta más habitual entre los agresores, tanto para chicos como chicas. Tal y como indicaron los alumnos consultados, y los resultados de otros estudios realizados sobre el tema, algunas de las múltiples maneras mediante las cuáles se manifiesta el ciberacoso son las amenazas y burlas e, igualmente, la suplantación de la identidad y el envío de material con contenido sexual (Calvete, Orue, Estévez, Villardón, y Padilla, 2010; Garaigordobil y Aliri, 2013; Durán-Segura y Martínez-Pecino, 2015). De la misma forma, la difusión de información personal o de rumores, fue un hecho que también sufrió un porcentaje significativo de hombres y mujeres de nuestro estudio. Como consecuencia de ello, fue bastante frecuente tanto el aislamiento social por parte de los jóvenes ciberacosados y la exclusión en redes sociales.

Los resultados del estudio aportan evidencia empírica con relación a algunas diferencias halladas entre lo expresado por chicos y chicas participantes, como son las siguientes:

- A la hora de establecer un perfil de persona acosada, existen diferencias entre hombres y mujeres respecto a la edad, ya que los hombres comenzaron a sufrir ciberacoso escolar entre los 13 y los 15 años, mientras que en las mujeres esa edad oscila entre los 16 y 17 años.

- La mayoría de las chicas, que sufrieron ciberacoso en el Instituto, recurrieron al aislamiento social, a diferencia de los chicos, quienes lo hicieron en menor medida, excluyéndose de las redes sociales principalmente.

- La mayoría de las mujeres consultadas en nuestro estudio indicaron que, cuando las ciberacosaron, respondieron a los acosadores por el mismo medio (chat, whatsapp, redes sociales) y han llorado, comunicándoselo a sus amigos. Sin embargo, esa comunicación no fue tan fluida cuando se trató de padres o docentes. En cuanto a los hombres consultados, mayoritariamente indicaron que han llorado y se lo han comunicado a los docentes.

- De los participantes que indicaron que ejercieron ciberacoso escolar durante su etapa en el Instituto, existen diferencias entre hombres y mujeres respecto a la edad, ya que los hombres empezaron a ejercerlo de forma mayoritaria entre los 
13 y los 15 años, mientras que en las mujeres esa edad oscila entre los 16 y los 17 años.

- Con relación a las víctimas mujeres, que han sufrido ciberacoso, indicaron que tuvieron entre sus agresores a antiguos amigos/as mientras que, en el caso de los hombres, los agresores indican que nunca habían sido amigos de las víctimas.

- Con relación a la actuación que los participantes recuerdan que se llevaba a cabo en su Instituto ante casos de ciberacoso, la mayoría de los que pertenecieron a centros públicos indicaron que se sancionaba al alumno acosador o que se ignoraba el hecho sucedido, mientras que los del centro privado se decantaron por la opción de la sanción de manera mayoritaria.

Estos datos difieren de algunos estudios realizados, los cuales han arrojado en sus resultados que el sexo, la edad, el nivel educativo y el rendimiento académico son algunos de los variables influyentes que se consideran en el cyberbullying, Sobre el sexo, a diferencia de nuestro estudio, los resultados son contradictorios, pues mientras algunos autores no encuentran diferencias (Smith, Mahdavi, Carvalho, Fisher, Russel y Tippet, 2008; Álvarez, Núñez, Álvarez, Dobarro, Rodríguez y González, 2011), otros observan mayor tendencia de los chicos como "ciberagresores" (Li, 2006) y las chicas como cibervíctimas (Ybarra, Diener-West y Leaf, 2007; Ortega, Calmaestra y Mora-Merchán, 2007; Patchin y Hinduja, 2012; Schneider, O’Donnell, Stueve y Coulter, 2012).

La mayoría de jóvenes, que sufrieron acoso en el Instituto en nuestro estudio, reconocieron que no se sintieron motivados para acudir a clase en su momento, ni para desarrollar sus estudios de manera adecuada. Estos resultados coinciden con lo expuesto por Muñoz (2016), quien indicó que, en los centros escolares españoles, hay menores que presentan mucha dificultad de integración escolar y en el proceso de aprendizaje porque afirman tener miedo y sentirse indefensos ante la amenaza, la violencia y el dominio de su agresor.

\section{CONCLUSIONES}

La implementación de medidas educativas es necesaria para la prevención del ciberacoso en centros educativos, ya que el mismo se ha convertido en uno de los fenómenos socialmente más problemáticos en España, causando numerosos problemas a sus víctimas, como mayores niveles de síntomas depresivos (Nixom, 2014), mayores niveles de ansiedad (Sontag, Clemans, Graber y Lyndon, 2011) y menor autoestima (O`Brien y Moules, 2013), entre otros.

Sin duda alguna, el ciberacoso es una problemática que supone un gran desafío para directivos de Instituto, docentes y padres, ya que a menudo puede escaparse de los ojos de, incluso, el mejor observador, convirtiéndose en una lucha imposible de ganar. Por ello, consideramos importante las propuestas de actuación recomendadas 
por los alumnos participantes de nuestro estudio ante casos de ciberacoso, ya que los mismos se han podido ver involucrados en esta problemática:

- Formación del profesorado y familias para que estén atentos a los cambios repentinos en los adolescentes, manteniendo una relación de confianza con ellos para que, en caso de problemas, sepan a quienes pueden acudir.

- Trabajo coordinado entre escuela y familia, por lo que las administraciones educativas deben ofrecerles las herramientas legislativas y las indicaciones para la prevención e intervención efectivas en temas relacionados con el ciberacoso, estableciendo protocolos de actuación a nivel nacional/autonómico.

- Crear conciencia de las consecuencias que acarrea el ciberacoso y la promoción de habilidades sociales como la empatía o la asertividad.

- Formar al alumnado ante la importancia de cuidar la privacidad (emplear cuentas privadas en redes sociales, limitar el número de contactos, configurar opciones de privacidad, no compartir información sensible, etc.).

Consideramos, tal y como indican Garaigordobil, Martínez y Machimbarrena (2017), que en todos los centros escolares debe haber un protocolo de actuación para los casos de acoso escolar o ciberacoso, posibilitando debates sobre dilemas morales, y dirigiendo el potencial de los jóvenes para hacer frente a injusticias o conductas propias de estos fenómenos. Por lo tanto, el acoso y el ciberacoso deben formar parte de una estrategia más amplia de lucha integral contra la violencia contra la infancia que incluya también estas formas de violencia y que establezca medidas de prevención, detección y recuperación, con un enfoque siempre basado en el interés superior de los niños y niñas, sean éstos víctimas o agresores (Calamestra, Escorial, García, Del Moral, Perezo y Ubrich, 2016).

\section{REFERENCIAS}

Álvarez-García, D., Núñez, C., Dobarro, A., y Rodríguez, C. (2015). Risk factors associated with cybervictimization in adolescence. International Journal of Clinical and Health Psychology, 15, 226-235. doi: https://doi.org/10.1016/j. ijchp.2015.03.002

Avilés, J. M. (2006). Diferencias de atribución causal en el bullying entre sus protagonistas. Electronic Journal of Research in Educational Psychology, 4(2), 201-209.
Bisquerra, R. (Coord.) (2004). Métodos de investigación educativa. Madrid: La Muralla.

Calmaestra, J., Escorial, A., García, P., Del Moral, C., Perazzo, C., y Ubrich, T. (2016). Yo a eso nojuego. Bullying, Ciberbullying y factores asociados. Madrid: Fundación Save The Children.

Calvete, E., Orue, L., Estévez, A., Villardón, L., y Padilla, P. (2010). Cyberbullying in adolescents: Modalities and aggressors' profile. Computers in Human Behavior, 26, 1128-1135. doi: https://doi. org/10.1016/j.chb.2010.03.017 
Cerezo, F., Arnaiz, P., Giménez, A., y Maquilón, J. (2016). Conductas de ciberadicción y experiencias de cyberbullying entre adolescentes de la Región de Murcia (España). Anales de Psicología, 32(3), 761,769. doi: http:// dx.doi.org/10.6018/analesps.32.3.217461

Del Rey, R., Mora-Merchán, J., Casas, J., Ortega-Ruiz, R., y Elipe, P. (2018). Programa "Asegúrate": Efectos en ciberagresión y sus factores de riesgo". Revista Comunicar, 56, 39-48. doi: https://doi.org/10.3916/C56-2018-04

Durán-Segura, M., y Martínez-Pecino, R. (2015). Ciberacoso mediante teléfono móvil e Internet en las relaciones de noviazgo entre jóvenes. Revista Comunicar, 44(22), 159-167. doi: http:// dx.doi.org/10.3916/C44-2015-17

Expósito, E., Navarro, E., Thoilliez, B., y López, E. (2010). Determinants of child well-being: A perspective from students of education. Comunicación presentada en la European Conference on Educational Research (ECER), Helsinki: Finlandia.

Fundación Mutua Madrileña y Fundación Anar (2016). I Estudio sobre ciberbullying según los afectados [página web]. Recuperado de https://bit.ly/2CP1YOR

Garaigordobil, M., y Oñederra, J. A. (2010). La violencia entre iguales: Revisión teórica y estrategias de intervención. Madrid: Pirámide.

Garaigordobil, M. (2011). Prevalencia y consecuencias del cyberbullying: una revisión. International Journal of Psychology and Psychological Therapy, 11(2), 233-254.

Garaigordobil, M., y Aliri, J. (2013). Ambivalent Sexism Inventory: Standardization and normative data in a sample of the Basque Country. Behavioral Psychology / Psicologia Conductual 2(1), 173-186.

Garaigordobil, M. (2015). Cyberbullying in adolescents and youth in the Basque Country: Prevalence of cybervictims, cyberaggressors, and cyberobservers. Journal of Youth Studies, 18(5), 569-582. doi: http://dx.doi.org/10.1080/13676261 .2014 .992324

Garaigordobil, M., Martínez, V., y Machimbarrena, J. (2017). Intervención en el bullying y ciberbullying: evaluación del caso Martín. Revista de Psicología Clínica con Niños y Adolescentes, 4, 2532.

García, Ma. D., y García, F. (2015). Acoso escolar. Análisis del sistema educativo y consideraciones jurídicas. En $\mathrm{M}^{\mathrm{a}}$ Rivas Vallejo y $\mathrm{M}^{\mathrm{a}}$ García Valverde (Dirs.), Tratamiento Integral del Acoso (300321). Navarra: Arazandi.

Giménez-Guado, A., Arnaiz-Sánchez, P., Cerezo-Ramírez, F., y Prodócimo, E. (2018). Percepción de docentes y estudiantes sobre el ciberacoso. Estrategias de intervención en Primaria y Secundaria. Revista Comunicar, 56(26). doi: https://doi.org/10.3916/C56-201803

León, B., Felipe, E., Fajardo, F., y Gómez, T. (2012). Cyberbullying en una muestra de estudiantes de Educación Secundaria: Variables moduladoras y redes sociales. Electronic Journal of Research in Educational Psychology, 1o(2), 771-788.

Marchena, R. (2012). La interacción profesorado-alumnado y la convivencia en el aula. Convives, 2, 11-16.

Martínez, R. A. (2007). La investigación en la práctica educativa: Guía metodológica de investigación para el diagnóstico $y$ evaluación en los centros docentes. Madrid: Ministerio de Educación y Ciencia.

McMillan, J., y Schumager, S. (2005). Investigación educativa. Madrid: Pearson Addison Wesley.

Moral, G. del, Suárez, C., Villarreal, M. ${ }^{\text {a E., }}$ y Musitu, G. (2014). Types of aggressive victims in bullying situations at secondary school. Infancia y Aprendizaje, 37(2), 
399-428. doi: https://doi.org/10.1080/o 2103702.2014.918816

Muñoz, J. (2016). Factores de riesgo en el acoso escolar y el ciberacoso: implicaciones educativas y respuesta penal en el ordenamiento jurídico español. Revista Criminalidad, 58(3), 71-86.

Navarro, R., Ruiz-Oliva, R., Larrañaga, E., y Yubero, S. (2015). The impact of cyberbullying and social bullying on optimism, global and school-related happiness and life satisfaction among 10-12-year-old schoolchildren. Applied Research in Quality of Life, 1O(1), 15-36. doi: https://doi.org/10.1007/s11482-0139292-0

O’Brien, N., y Moules, T. (2013). Not sticks and stones but tweets and texts: Findings from a national cyberbullying project. Pastoral Care in Education. International Journal of Psychology in Education, 31(1), 53-65. doi: https://doi.org/10.1080 02643944.2012.747553

Ortega, R., Calmaestra, J., y Mora-Merchán, J. A. (2007). Cuestionario cyberbullying [página web]. Recuperado de https:// www.uco.es/laecovi/img/recursos/ RFUY4MDDVCZWHkm.pdf

Palacios, V., Polo, M., Felipe, E., León, B., y Fajardo, F. (2013). Tipología familiar y dinámica bullying/ciberbullying en educación secundaria. European Journal of Investigation in Health, Psichology and Education, 3(2), 161-170.

Patchin, J. W., e Hinduja, S. (2012). Cyberbullying. An update and synthesis of the research. En J. W. Patchin y S. Hinduja (Eds.), Cyberbullying prevention and response. Expert perspectives (13-35). New York: Routledge.

Perren, S., Dooley, J., Shaw, T., y Cross, D. (2010). Bullying in school and cyberspace: Associations with depressive symptoms in Swiss and Australian adolescents. Child and Adolescent Psychiatry and Mental Health, 4(28), 28-38. doi: https://doi. org/10.1186/1753-2000-4-28
Rodríguez-Hidalgo, A. J., Ortega-Ruiz, R., y Zych, I. (2014). Victimización étnico-cultural entre iguales: Autoestima y relaciones en la escuela entre diferentes grupos culturales de estudiantes en Andalucía (España). Revista de Psicodidáctica, 19(1), 191210. doi: https://doi.org/10.1387/ RevPsicodidact.7909

Slonje, R., Smith, P. K., y Frisén, A. (2013). The nature of cyberbullying, and strategies for prevention. Computers in Human Behavior, 29, 26-32. doi: https:// doi.org/10.1016/j.chb.2012.05.024

Sontag, L. M., Clemans, K. H., Graber, J. A., y Lyndon, S. T. (2011). Traditional and cyber aggressors and victims: a comparison of psychosocial characteristics. Journal of Youth and Adolescence, 40(4), 392-404. doi: https://doi.org/10.1007/s10964010-9575-9

Sourander, A., BrunsteinKlomek, A. B., Ikonen, M., Lindroos, J., Luntamo, T., Koskelainen, M., Ristkari, T., y Helenius, H. (2010). Psychosocial risk factors associated with cyberbullying among adolescents. Archives of General Psychiatry, 67(7), 720728. doi: https://doi.org/10.1001/ archgenpsychiatry.2010.79

Smith, P. K., Mahdavi, J., Carvalho, M., Fisher, S., Russel, S., y Tippet, N. (2008). Cyberbullying: its nature and impact in secondary school pupils. Journal of Child Psychology and Psychiatry, 49(4), 376385. doi: https://doi.org/10.1111/j.14697610.2007.01846.x

Schneider, S. K., O’Donnell, L., Stueve, A., y Coulter, R. W. S. (2012). Cyberbullying, school bullying, and psychological distress: A regional census of high school students. American Journal of Public Health, 102(1), 171-177. doi: https://doi. org/10.2105/AJPH.2011.300308

Wolke, D., y Lereya, S. T. (2015). Longterm effects of bullying. Archives of Disease in Childhood, 10o(9), 879- 
885. doi: https://doi.org/10.1136/ archdischild-2014-306667

Ybarra, M. L., Diener-West, M., y Leaf, P. J. (2007). Examining the overlap in internet harassment and school bullying: Implications for school intervention. Journal of Adolescent Health, 41(6), 42-50. doi: https://doi.org/10.1016/j. jadohealth.2007.09.004

Yubero, S., Larrañaga, E., Navarro, R. y Elche, M. (2018) Padres, hijos e Internet. Socialización familiar de la red. Universitas Psiychologica, 17(2), faltan páginas. doi: http://dx.doi.org/10.11144/ javeriana.upsy17-2.phis
Zwierzynska, K., Wolke, D., y Lereya, T. S. (2013). Peer victimization in childhood and internalizing problems in adolescence: A prospective longitudinal study. Journal of Abnormal Child Psychology, 41(2), 309-323. doi: https://doi.org/10.1007/ s10802-012-9678-8

Zych, I., Ortega-Ruiz, R., y Marín-López, I. (2016). Cyberbullying: a systematic review of research, its prevalence and assessment issues in Spanish studies. Psicología Educativa, 22(1), 5-18. doi: https://doi. org/10.1016/j.pse.2016.03.002

\section{PERFIL ACADÉMICO Y PROFESIONAL DE LOS AUTORES}

Juan Carlos Rivadulla López. Doctor en Educación por la Universidade da Coruña (UDC). Profesor Ayudante Doctor en los Grados en Educación Infantil y Primaria y en el Máster de Didácticas Específicas en la Facultade de Ciencias da Educación de la UDC.

E-mail: juan.rivadulla@udc.es

Dirección:

Facultade de Ciencias da Educación,

Campus de Elviña, $\mathrm{s} / \mathrm{n}$,

C.P. 15071. A Coruña.

Marisol Rodríguez Correa. Doctora en Pedagogía por la Universidade da Coruña (UDC). Profesora asociada en la Universidad Internacional de la Rioja en el Master Universitario en Formación de Profesorado de Educación Secundaria. Sus líneas de investigación están centradas en el análisis de la integración de las TIC en la enseñanza y la innovación y la mejora de las instituciones educativas.

E-mail: marisol.rodriguez@unir.net

Dirección:

Calle Islas Cíes. N 3.2A.

C.P. 15190. A Coruña.

Fecha de recepción del artículo: 00/00/2019

Fecha de aceptación del artículo: 00/00/2019

Fecha de aprobación para maquetación: 00/00/2019 


\section{ANEXO}

El presente cuestionario forma parte de una investigación para conocer cómo se utilizan las nuevas tecnologías (Teléfono Móviles e Internet) en las relaciones entre los y las jóvenes como tú. Por favor responde de manera sincera a las preguntas, ya que el cuestionario es anónimo y nadie sabrá cuáles son tus respuestas.

Gracias por tu sinceridad y por dedicarnos tu tiempo.

\section{DATOS DE IDENTIFICACIÓN}

1. Sexo
a. Hombre

2. Edad

b. Mujer

3. El Instituto donde estudiaste era ¿̇público o privado?

4. Rama de conocimiento de los estudios que cursas:

5. Lugar de residencia:

6. ¿Tienes teléfono móvil? Sí No

7. ¿Tienes acceso a Internet en casa? Sí No

8. ¿'Tienes acceso a Internet desde tu móvil? Sí No

El cyberbullying es un tipo de molestia o acoso en que se utilizan medios tecnológicos para meterse con alguien como, por ejemplo, el móvil o Internet. Las preguntas que queremos que respondas a continuación se refirieren a tu época de estudiante en el Colegio/Instituto (primaria, ESO o Bachillerato)

\section{a. Casos de ciberacoso observados en el Instituto}

9. ¿̇Ha sucedido este fenómeno cuando estudiaste en el Colegio/Instituto (tú no eras la víctima)?
a. No, no hubo cyberbullying en mi Centro.
b. Sólo se produjo una o dos veces.
c. Sí, alrededor de una vez a la semana.
d. Sí, varias veces a la semana.

\section{b. Perfil de los participantes que admiten haber sufrido ciberacoso en el Instituto}

10. ¿̇A qué edad comenzaron a acosarte?

11. ¿Cuántas veces se han metido contigo o te han acosado a través de tú móvil o del Internet en el Colegio/Instituto?

a. Ninguna, no ha sucedido.

b. Sólo se produjo una o dos veces. 

c. Sí, alrededor de una vez a la semana.
d. Sí, Varias veces a la semana.

12. ¿En qué consistía el acoso?

13. ¿Qué hacías normalmente cuando alguien se metía contigo o te acosaba a través del móvil o del Internet? (Para esta pregunta puedes marcar más de una opción)

a. Nada, no me ha pasado nada de eso.

b. Me he puesto a llorar.

c. He apagado el móvil o el ordenador.

d. He ignorado lo que estaba pasando.

e. Le he dicho que parase por el mismo medio (llamada, SMS, E-mail, chat, etc.).

f. Se lo he dicho a mis amigos.

g. Se lo he dicho a mis padres.

h. Se lo he dicho a mi tutor u otro profesor.

i. He intentado hacerles lo que ellos a mí.

14. En el período en el que sufrías ciberacoso, ¿cómo te sentías en el Instituto? ¿Qué grado de satisfacción escolar tenías?

\section{c. Perfil de los participantes que admiten haber ejercido ciberacoso en el Instituto}

15. ¿A qué edad comenzaste a acosar a compañeros/as?

16. ¿Te has metido tú con alguien o has acosado, a través del móvil o del Internet, a otras personas en el Colegio/Instituto?
a. Ninguna, no ha sucedido.
b. Sólo se produjo una o dos veces.
c. Sí, alrededor de una vez a la semana.
d. Sí, Varias veces a la semana.

17. ¿Qué grado de amistad te unía con la víctima?
a. No éramos amigos
b. Éramos amigos en el momento en el que se produjo el acoso
c. Habíamos sido amigos antes del acoso

18. ¿Cuántas personas participabais en el ciberacoso?

19. ¿Cuál era el motivo que os llevaba a ciberacosar a un/a compañero/a? 


\section{d. Medidas contra el ciberacoso}

20. ¿Consideras según tu experiencia que en tu Colegio/Instituto se empleó un protocolo de actuación ante casos de ciberbullying? (Para esta pregunta puedes marcar más de una opción)

a. Sí, se hacía un seguimiento y evaluación constante de las situaciones de acoso.

b. Sí, se sancionaba al alumno.

c. Sí, se reforzaba la vigilancia en zonas (pasillos, recreos, salida).

d. Sí, hablábamos sobre el tema en clase.

e. Sí, se hablaba con la familia del alumno acosador y el alumno acosado.

f. Sí, se sensibilizaba a los alumnos sobre el fenómeno del acoso escolar con charlas o proyectos educativos.

g. No, se ignoraba el hecho sucedido.

21. ¿Cómo crees que se podrían evitar situaciones de ciberbullying en los niveles de ESO o bachillerato?

\section{MUCHAS GRACIAS POR TU PARTICIPACIÓN}

